\title{
Estimating models of inverse systems
}

Ylva Jung and Martin Enqvist

\section{Linköping University Post Print}

\section{Tweet}

N.B.: When citing this work, cite the original article.

Original Publication:

Ylva Jung and Martin Enqvist, Estimating models of inverse systems, 2013, Proceedings of the 52nd IEEE Conference on Decision and Control, 7143-7148.

(C2013 IEEE. Personal use of this material is permitted. However, permission to reprint/republish this material for advertising or promotional purposes or for creating new collective works for resale or redistribution to servers or lists, or to reuse any copyrighted component of this work in other works must be obtained from the IEEE.

http://www.ieee.org

Postprint available at: Linköping University Electronic Press

http://urn.kb.se/resolve?urn=urn:nbn:se:liu:diva-103999 


\title{
Estimating models of inverse systems
}

\author{
Ylva Jung and Martin Enqvist
}

\begin{abstract}
This paper considers the problem of how to estimate a model of the inverse of a system. The use of inverse systems can be found in many applications, such as feedforward control and power amplifier predistortion. The inverse model is here estimated with the purpose of using it in cascade with the system itself, as an inverter. A good inverse model in this setting would be one that, when used in series with the original system, reconstructs the original input. The goal here is to select suitable inputs, experimental conditions and loss functions to obtain a good input estimate. Both linear and nonlinear systems will be discussed.

For nonlinear systems, one way to obtain a linearizing prefilter is by Hirschorn's algorithm. It is here shown how to extend this to the postdistortion case, and some formulations of how the pre- or postinverter could be estimated are also presented.
\end{abstract}

\section{INTRODUCTION}

The behavior of a system $S$ can be modified in a multitude of ways. In automatic control, the main choices are feedforward and feedback control. Feedback can handle phenomena like disturbances, model uncertainties and unstable systems, but a bad feedback loop may cause instability. Feedforward control does not need any measurements but ideal feedforward control (using $F_{f}=S^{-1}$ ) requires perfect knowledge of the system (as well as all disturbances) and that both $S$ and $S^{-1}$ are stable. These are of course limiting assumptions. A benefit with feedforward is that two cascaded stable systems will always be stable. Often, both feedforward and feedback control are used to get the advantages of both approaches.

In some cases it is however not possible or desirable to use feedback. One application example of this is power amplifiers in communication devices, which are often nonlinear and/or dynamic, causing interference in adjacent transmitting channels [1]. To be useful, linearization is needed. However, one does not want to work with the amplified signal, but rather the input signal to the system. A prefilter that inverts the nonlinearities/dynamics, called a predistorter, is thus preferable. In sensor applications it is rather a postdistortion that is needed. If the sensor itself has dynamics or a nonlinear behavior, the sensor output is not the true signal but will also contain some sensor contamination. This would have to be handled at the sensor output.

In the above applications, finding the inverse is a crucial point. The inverse model is here estimated with the purpose of using it in cascade with the system itself, see Fig. 1. This inverse model can either be built on physical knowledge, or

This work was partially supported by the Excellence Center at LinköpingLund in Information Technology (ELLIIT), and the Center for Industrial Information Technology at Linköping University (CENIIT).

Y. Jung and M. Enqvist are with the Department of Electrical Engineering, Linköping University, SE-581 83 Linköping, Sweden, phone: +46(0)13284474, e-mail: ylvju@isy.liu.se, maren@isy.liu.se.

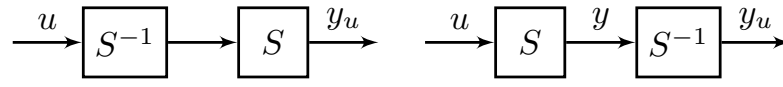

Fig. 1. The intended use of the estimated inverses. The left figure shows predistortion, where the inverse $S^{-1}$ is applied before the system $S$, and the right shows postdistortion, where the order is reversed.

it can be estimated based on measured data. Each application entails restrictions and special conditions to attend to.

In system identification, one should usually estimate the system in the setting it should be used in, concerning for example the choice of input and the experimental conditions [2, Ch. 13], [3] and [4]. It is important to choose the input signal to capture the desired characteristics of the system, but what does that correspond to in this case? Another important topic in system identification is the choice of loss function. It should reflect the goal of the identification, and different properties of the system can be emphasized. In this setup, we want to use these degrees of freedom and the flexibility of the model to obtain a good input estimate.

The contribution in this paper is two-parted. One is the investigation of the loss function for the estimation of the inverse, and the adjoining discussion on the choice of inverse estimation approach. This concerns the two approaches forward estimation followed by inversion, and the estimation of the inverse system directly, for linear time invariant (LTI) dynamical systems. The other concerns Hirschorn's method [5] and how it can be rewritten into a postdistortion method, as well as the formulation of the different settings under which this pre- or postinverter could be estimated.

The disposition of the paper is as follows. Different approaches to the inversion and estimation of inverse systems are presented in Section II. In Section III the identification of LTI dynamical systems is discussed, followed by an example in Section IV. Section V shortly introduces inversion techniques for nonlinear systems and in Section VI, Hirschorn's method (a predistortion method), its extension to postdistorter and some aspects of the estimation of this distorter are presented. Conclusions are in Section VII.

\section{System InVERSE Estimation}

In system identification, the goal is to achieve as good a model as possible to explain the behavior of $y$ by a prediction or simulation $\hat{y}(t \mid \theta)$, which depends on estimated model parameters and the input $u$. This is done using measured data, usually input data, $u(t)$, and output data, $y(t)$. Here, a model describing the system itself will be referred to as a forward model and a model describing the inverse will be called an inverse model. 
TABLE I

THE INPUTS AND OUTPUTS TO THE IDENTIFICATION PROCEDURES

\begin{tabular}{|c|c|c|c|c|}
\hline Method & Input & Output & Requires & Model \\
\hline A & $u$ & $y$ & & forward \\
\hline B & $u$ & $u$ & $\hat{S}$ & inverse \\
\hline C & $y$ & $u$ & & inverse \\
\hline
\end{tabular}

The inverse model is estimated with the purpose of using it in series with the system itself, as an inverter, see Fig. 1. The goal is to minimize the difference between the input $u$ and the output from the cascaded systems, $y_{u}$. A good model in this setting is one that, when used in series with the original system, regains the original input, so that $y_{u}=u$.

There are three main approaches to the estimation of an inverse of a system $S$, described in more detail below.

A) In a first step, the forward model $\hat{S}$ is estimated in the standard way (with input data $u$ and output data $y$ ). Step two is to invert the resulting model to obtain an estimated inverse $\hat{S}^{-1}$.

B) In a pre-step, the forward model $\hat{S}$ is estimated in the standard way (with input data $u$ and output data $y$ ). This model is used in series with an inverse model, $\widehat{S^{-1}}$, and the inverse model parameters are estimated in this setting, by minimizing the difference between the input $u$ and the simulated, distorted output $y_{u}$.

C) The identification is done in one step, by identifying the inverse $\widehat{S^{-1}}$ directly, using input data $y$ and output data $u$.

The inputs and outputs used are summarized in Table I.

The identification in Method A is the standard one, as described in for example [2] and [4], and the inversion is discussed in [6] in the feedforward control application. The use of feedforward control based on an inverse model of the system in the presence of plant uncertainty is discussed in [7]. A good thing is that the identification uses standard methods, but on the other hand, an inversion is required, and the weighting of the model fit is not necessarily optimal for the use intended here.

Method B is often used in power amplifier predistortion [8], [9], [10], and is then also called Direct Learning Control (DLA). The quality of the inverse and the forward models are closely coupled, and two choices are available. It is often preferable to obtain a simple inverse model, as in the predistorter case, and this restriction can also be applied to the forward model. Alternatively, a more complex forward model can be used to make sure that as much as possible of the system behavior is captured, while keeping the inverse model less complex. The choice comes down to the implementation - if the forward model has to be implemented, also this model needs to have a limited complexity. A good thing with this approach is that the estimation of the inverse is done with no noise present, but it also requires two (possibly nonconvex) minimizations (with the risk of obtaining local minima), and the quality of the inverse clearly depends on the quality of the forward model.
Method $\mathrm{C}$ is also called Indirect Learning Control (ILA) in power amplifier (PA) predistortion applications and is evaluated for PA predistortion in [9] and [10]. This approach assumes that the predistorter and the postdistorter are interchangeable (commutativity). An advantage with this method is that the inverse is estimated in the setting it is going to be used, and that the weighting is possibly better than for the first method, but the noise entering at the input risks causing a biased estimate.

In PA predistortion applications, the third approach (ILA) is more commonly used than the second (DLA) [10]. In [10], comparisons performed indicate that the DLA performs better in the simulation setup used, whereas in [9] the ILA seems to perform slightly better.

Inverse systems entail a number of problems. Any nonminimum-phase zeros of the original system will become unstable poles of the inverse system. However, if a delay can be allowed, a stable approximative filter can often be found [11]. If the transfer function is strictly proper, the amplification will approach zero at high frequencies. The inverse of a strictly proper system will be improper, that is, high frequency contents will be amplified. The amplification of high frequency noise should be avoided, as is described in [12]. Here, only the case when the system and its inverse are both stable and causal will be investigated.

\section{INVERSE IDENTIFICATION OF LTI SYSTEMS}

To simplify the discussion, we will start by looking at LTI dynamical systems. The model estimation is done in open loop and under the assumption that the output was created according to

$$
y(t)=G_{0}(q) u(t)+H_{0}(q) e_{0}(t)
$$

where $G_{0}$ is the true system, $H_{0}$ is the true noise dynamics and $e_{0}$ is a white noise sequence. In system identification, the goal is often to find the minimizing argument of a function of the prediction error $\varepsilon(t, \theta)$

$$
\begin{aligned}
\hat{\theta} & =\underset{\theta}{\arg \min } \frac{1}{N} \sum_{t=1}^{N} \varepsilon(t, \theta)^{2} \\
& =\underset{\theta}{\arg \min } \frac{1}{N} \sum_{t=1}^{N}[y(t)-\hat{y}(t \mid \theta)]^{2},
\end{aligned}
$$

where $y(t)$ is the measured output and $\hat{y}(t \mid \theta)$ is the predicted output, given the model parameters $\theta$. Here, we use a fixed noise model $H_{*} \equiv 1$ such that the prediction is described by $\hat{y}(t \mid \theta)=G(q, \theta) u(t)$. Looking at the identification from a frequency domain point of view, the minimization criterion in (2) can asymptotically be written as [2, (8.71)]

$$
\hat{\theta}=\underset{\theta}{\arg \min } \int_{-\pi}^{\pi}\left|G_{0}\left(e^{i \omega}\right)-G\left(e^{i \omega}, \theta\right)\right|^{2} \Phi_{u}(\omega) \mathrm{d} \omega
$$

where $G\left(e^{i \omega}, \theta\right)$ is the model and $\Phi_{u}(\omega)$ is the spectrum of the input signal. The estimation will thus be done in a way to emphasize the model fit in frequency bands where the transfer function and the input spectrum are large enough to have a significant impact on the total criterion. The 
minimization is done with respect to the product of the model fit $\left(\left|G_{0}-G\right|^{2}\right)$ and the input spectrum. If the input is white noise, it is thus more important to obtain a good model fit at frequencies with a large transfer function magnitude.

If instead the goal is to estimate the inverse model to be used as described in Section II, the minimization criterion in the time domain can be written

$$
\hat{\theta}=\underset{\theta}{\arg \min } \frac{1}{N} \sum_{t=1}^{N}\left[u(t)-\frac{1}{G(q, \theta)} y(t)\right]^{2} .
$$

The frequency domain equivalent to (4), when $y$ is noisefree, is

$$
\hat{\theta}=\underset{\theta}{\arg \min } V_{\text {inv }}(\theta)
$$

where the loss function is

$$
\begin{aligned}
& V_{\text {inv }}(\theta)=\int_{-\pi}^{\pi}\left|\frac{1}{G_{0}\left(e^{i \omega}\right)}-\frac{1}{G\left(e^{i \omega}, \theta\right)}\right|^{2} \Phi_{y}(\omega) \mathrm{d} \omega \\
& =\int_{-\pi}^{\pi}\left|\frac{1}{G_{0}\left(e^{i \omega}\right)}-\frac{1}{G\left(e^{i \omega}, \theta\right)}\right|^{2}\left|G_{0}\left(e^{i \omega}\right)\right|^{2} \Phi_{u}(\omega) \mathrm{d} \omega \\
& =\int_{-\pi}^{\pi}\left|1-\frac{G_{0}\left(e^{i \omega}\right)}{G\left(e^{i \omega}, \theta\right)}\right|^{2} \Phi_{u}(\omega) \mathrm{d} \omega \\
& =\int_{-\pi}^{\pi}\left|G\left(e^{i \omega}, \theta\right)-G_{0}\left(e^{i \omega}\right)\right|^{2} \frac{\Phi_{u}(\omega)}{\left|G\left(e^{i \omega}, \theta\right)\right|^{2}} \mathrm{~d} \omega
\end{aligned}
$$

using $\Phi_{y}=\left|G_{0}\left(e^{i \omega}\right)\right|^{2} \Phi_{u}$ with no noise present. The minimization in (7) is similar to the weighting for the input error case where $H=G$ so that $y(t)=G u+G e=G(u+e)$, that is, the error enters the system with the input [13].

Comparing the minimization criterion for the forward estimation in (3) to the one for the inverse estimation in (6), the weighting is clearly different. In the forward case, a relative model error at a frequency where the system amplification is small, will affect the criterion much less than a model error at a frequency where the system amplification is large. In the inverse estimation case, though, a relative model error will have the same effect on the criterion, regardless of the amplification at that frequency, for frequencies with the same input spectral density. The weighting, and the model fit, between the different frequencies will thus be shifted to better reflect the importance of a good fit also at frequencies with a small transfer function magnification.

The time domain criterion (4) thus leads to the frequency domain description (6), and the weighting is automatically done to match the usage of the inverse model estimate.

\section{An Illustrative Linear DyNAmic EXAMPLE}

Let us look at a small example. The goal is to obtain a system inverse to be used in series with the original system, in order to retrieve the input, see Fig. 1 (left). The input $u$ and the noise-free output $y$ are measured. The system has two resonance frequencies, at $\omega=1 \mathrm{rad} / \mathrm{s}$ and $\omega=10 \mathrm{rad} / \mathrm{s}$. The magnitudes of the two resonance peaks are very different, with the first one a hundred times larger than the second one. The true system $G_{0}$ is described by

$$
G_{0}(s)=\frac{10}{s^{4}+1.1 s^{3}+101.1 s^{2}+11 s+100}
$$

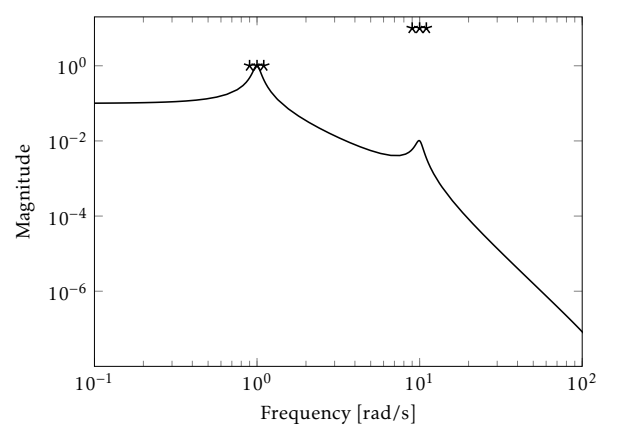

Fig. 2. Bode magnitude plot of $G_{0}$ in (8) (solid line). The stars mark the amplitude of the multisine input ( $u$ in (9)) components at each frequency.

and the Bode magnitude diagram is shown in Fig. 2. The input consists of three sinusoids around each of the two resonance frequencies such that the input power is concentrated in two bands, centered around these resonance frequencies,

$$
u=\sum_{k=1}^{6} a_{k} \sin \left(\omega_{k} t+\phi_{k}\right)
$$

with $a_{k}=1$ for $k=1,2,3, a_{k}=10$ for $k=4,5,6$, $\omega_{k}=\{0.9,1,1.1,9,10,11\}$ and $\phi \sim U[-\pi \pi]$. The input amplitude is illustrated by the stars in Fig. 2. The sampling time is $T_{s}=0.02 \mathrm{~s}$ and $N=10000$ simulated measurements have been collected.

With the goal of using an FIR model as prefilter to recover the input $u$, two models have been estimated, using Methods A and C in Section II. First, a forward model has been estimated as an output error (OE) model using System Identification ToolBox in MATLAB, with [nb nf nk] $=\left[\begin{array}{lll}1 & 3 & 0\end{array}\right]$. This model has then been inverted resulting in an FIR model with 4 terms. The approximate inverse using Method $\mathrm{C}$ is an FIR model with 4 terms, i.e. [nb $\mathrm{nf} n k$ ] = $\left[\begin{array}{lll}4 & 0 & 0\end{array}\right]$, and will have a very different weighting. Hence, the two inverses will catch different properties of the system.

As can be seen in the Bode magnitude plot in Fig. 3, the Method A model has a much better fit around $\omega=1 \mathrm{rad} / \mathrm{s}$ and almost perfectly models the resonance peak, but completely misses the second resonance peak at $\omega=10 \mathrm{rad} / \mathrm{s}$. The Method $\mathrm{C}$ model does not manage to catch either of the resonance peaks in a satisfactory way but catches both of the resonance frequencies. That is, the amplification at $\omega=1$ and $10 \mathrm{rad} / \mathrm{s}$ is well captured, but not the resonance peaks around. Estimating the forward model in the standard way will clearly focus on the frequencies where the product of model fit $\left(\left|G_{0}-G\right|^{2}\right)$ and input spectrum is large. When this system approximation is then inverted, the errors around $\omega=10 \mathrm{rad} / \mathrm{s}$ will become prominent.

In the time domain plot in Fig. 4, it is clear that the inverse model estimated directly better reconstructs the input than the inverted forward model. In Fig. 5, the periodograms of the reconstructed inputs are shown. At the lower frequency around $\omega=1 \mathrm{rad} / \mathrm{s}$, the Method A model captures the input almost perfectly, but around $\omega=10 \mathrm{rad} / \mathrm{s}$, the reverse is true and the Method $\mathrm{C}$ model performs better. 


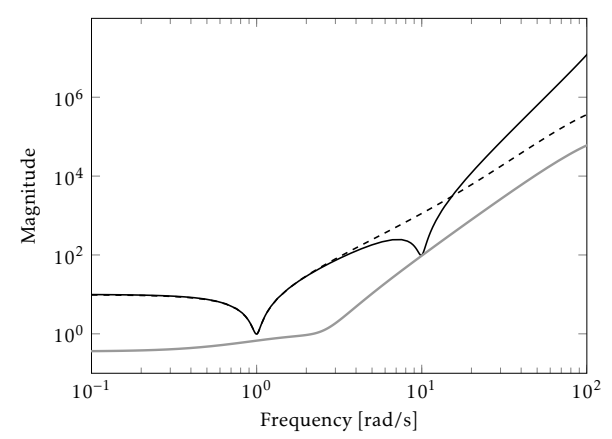

Fig. 3. Bode magnitude response of $G_{0}^{-1}$ (black solid line) and models using Methods A (black dashed line) and C (gray solid line). The inverted forward model perfectly catches the resonance peak at $\omega=1 \mathrm{rad} / \mathrm{s}$, but completely misses the second peak. The inverse estimated directly does not catch either of the resonance peaks in a satisfactory way, but instead has an accurate modeling of both peak frequencies.

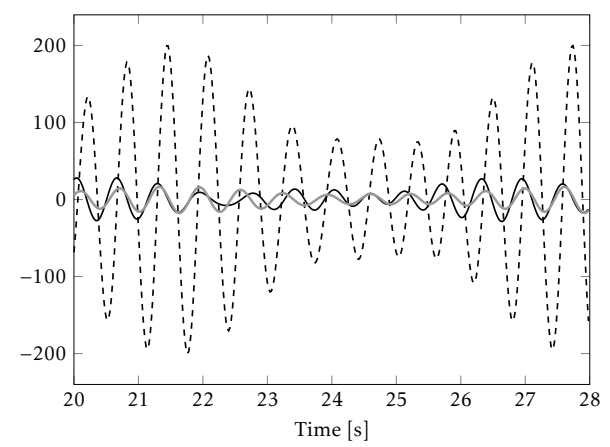

Fig. 4. The input $u$ (black solid line), and the reconstructed input $y_{u}$ using the Methods A (black dashed line) and C (gray solid line). The estimation of the inverse cannot perfectly reconstruct the input, but is clearly better than the inverted forward model.
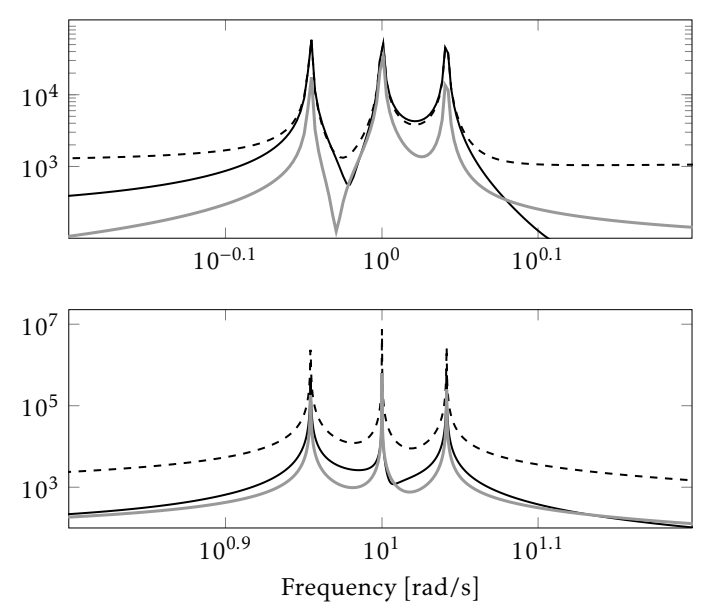

Fig. 5. Periodogram of the input $u$ (black solid line), and the reconstructed input $y_{u}$ using the Methods A (black dashed line) and C (gray solid line) around $\omega=1 \mathrm{rad} / \mathrm{s}$ (top) and $\omega=10 \mathrm{rad} / \mathrm{s}$ (bottom). It is clear also in the frequency domain that the forward model better captures the behavior around $\omega=1 \mathrm{rad} / \mathrm{s}$ than the inverse estimation, but the reverse is true at $\omega=10 \mathrm{rad} / \mathrm{s}$.
As shown in this small example, there are clearly occasions when it is advantageous to estimate an approximate inverse directly as opposed to estimating the forward model and then inverting it.

\section{NONLINEAR INVERSION}

The inversion of a nonlinear system is nontrivial, and different approaches have been used to cope with this. In [14, p 51], the nonlinear system $S$ is divided into a linear part, $L$, and a nonlinear part, $N$. It is shown that the inverse of $S$ can be obtained in a feedback loop with the nonlinear part $N$ in the feedback and the linear inverse $L^{-1}$ in the forward path. It follows that the nonlinear part $N$ does not have to be inverted, and that only the linear part $L$ is to be inverted.

Volterra series are also used to model nonlinear dynamical systems, and it can be shown that the $p$ th order postinverse equals the $p$ th order preinverse, so that the predistorter and postdistorter are interchangeable [15]. The drawback with Volterra series is that they are computationally very heavy, with many parameters to estimate.

Hammerstein systems are often used as a forward model in PA predistortion. The nonlinear compensation can be either a Wiener system [9] or a second Hammerstein system. This structure can also be extended with multiple branches, called parallel Hammerstein.

\section{HIRSCHORN'S METHOD}

A feedback solution for nonlinear systems is the exact input-output linearization that makes use of a known model of the system to obtain overall linear dynamics, determined by the user. In exact linearization (also called input-output linearization) [16], the output from a nonlinear system $S$,

$$
\begin{aligned}
\dot{x} & =f(x)+g(x) u \\
y & =h(x)
\end{aligned}
$$

is differentiated w.r.t. time, to obtain a relation between the differentiated output $y^{(\gamma)}$ and the input, $u$. The input

$$
u=\alpha(x)+\beta(x) r
$$

can be chosen so that it leads to a direct relation between the differentiated output $y^{(\gamma)}$ and the reference $r, y^{(\gamma)}=r$. This now describes a system with linear dynamics, and the nonlinear feedback loop can be combined with linear theory to obtain the desired dynamics, denoted $G_{m}$. The overall system from $r$ to $y$ (the nonlinear system with the nonlinear and linear feedbacks) will thus be linear, and the dynamics described by the transfer function $G_{m}$, chosen by the user.

Exact linearization requires the knowledge of all the states, and is therefore often used in combination with a nonlinear observer. This can lead to a complicated feedback loop. Here, it is assumed that any zero dynamics present are stable.

\section{A. Preinversion}

In Hirschorn's method, exact linearization is used to construct a linear system [5]. Given a good enough model, it should be possible to use the model not only in the feedback (in the construction of $u$ in (11)) but also as a simulation 


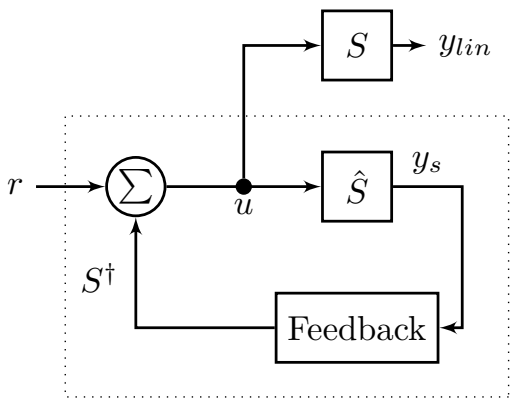

Fig. 6. Block diagram of Hirschorn's method, where the system $S$ is replaced by a model $\hat{S}$ in the exact linearization feedback loop. The input signal calculated in this way is then also applied to the real system $S$. The simulation system and feedback loop that leads to an overall linear behavior between $r$ and $y_{s}$ is denoted $S^{\dagger}$. The input to $S^{\dagger}$ is the reference $r$ and the output is the control signal $u$.

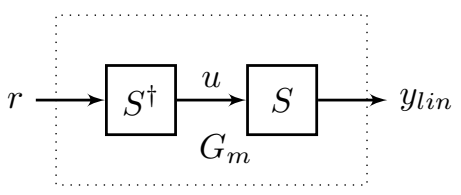

Fig. 7. The predistortion block $S^{\dagger}$ obtained using Hirschorn's method in series with the real system leads to an overall linear behavior between $r$ and $y_{l i n}$.

model. If the output from the simulated model is fed back to the controller, see Fig. 6, the overall system from reference $r$ to output $y_{s}$ will be linear with the dynamics $G_{m}$. Also, the input calculated for this (simulated) system leads to the desired dynamics, and the same input signal can be used also for the true system. A pure feedforward controller is thus obtained, as in [5], see Fig. 7. This can also be seen as an observer with no measurement inputs.

\section{B. Postinversion}

Let the nonlinear system be denoted $S$, the precompensation $S^{\dagger}$ (since it is not really an inverse of $S$, but rather creates a system that, in series with $S$ will be linear) and the dynamics of the overall linear system $G_{m}$. The goal is to obtain a linear response to $u$ by using a postinverse on the output $y$. The above method can be seen as an inversion of the nonlinearities of the system - the output from the overall system will be linear with dynamics $G_{m}$, chosen by the user (assuming the model is accurate enough, of course). Here, preinverse and postinverse are not interchangeable; Hirschorn's method tells us only how to determine the input to the nonlinear system such that the reference-to-output has the linear dynamics $G_{m}$, not how to manipulate the output to make it a linear response to the input. If a postinverse is desired, a different setup is needed.

It is known that $S^{\dagger}$ in cascade with $S$ leads to a linear system $G_{m}$, so that $y=G_{m} r$ with $r$ the reference, cf. Fig. 7 . Assume that $u$ was actually created by a prefilter, $S^{\dagger}$, with $u$ as output and the fictitious signal $\tilde{r}$ as input, see Fig. 8. An estimate of this signal can then be obtained by

$$
\bar{r}=G_{m}^{-1} y
$$

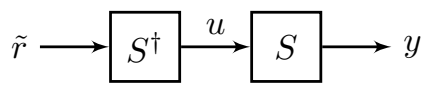

Fig. 8. The (possibly fictitious) reference signal $\tilde{r}$ can be seen as input to the block $S^{\dagger}$, creating the input $u$ to the nonlinear system $S$.

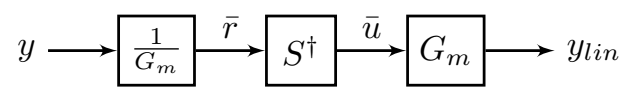

Fig. 9. Hirschorn's method used as postdistortion, when the output can be assumed to be created according to Fig. 8. The block $S^{\dagger}$ cannot simply be applied at the output $y$, but has to be manipulated to obtain a linear behavior between $u$ and $y_{l i n}$.

where, if no transients or noise are present, $\bar{r}=\tilde{r}$. An estimate of the input $u$, called $\bar{u}$, can then be obtained by filtering $\bar{r}$ by $S^{\dagger}$. Now, to obtain the desired dynamics, $\bar{u}$ must be filtered by the linear function $G_{m}$, see Fig. 9. The cascade of these three blocks $\left(1 / G_{m}, S^{\dagger}\right.$ and $\left.G_{m}\right)$, thus make up a postdistorter that leads to a linear response between $u$ (not available for manipulation) and $y_{l i n}$ in Figure 10.

Example - Hirschorn's Postinverse: Consider the nonlinear system

$$
\begin{aligned}
\dot{x}_{1} & =-x_{1}^{3}+x_{2}+w_{1} \\
\dot{x}_{2} & =-x_{2}+u+w_{2} \\
y & =x_{1}
\end{aligned}
$$

with process noise $w_{i} \in N(0,0.05)$ and a multisine input. The nonlinear feedback

$$
u=-3 x_{1}^{5}+3 x_{1}^{2} x_{2}+x_{2}+\tilde{u}
$$

leads to a linear system $\ddot{y}=\tilde{u}$. Now, linear theory can be applied and pole placement has been used to get an overall system response from reference $r$ to output $y$ corresponding to the one from $G_{m}(s)=1 /\left(s^{2}+5 s+6\right)$. The output from the nonlinear system (13) is plotted in Fig. 11 together with the output from the desired dynamics $G_{m}$.

A preinverse $S^{\dagger}$ has been constructed as in Fig. 6. $S^{\dagger}$ has been used as a preinverse, as well as a postinverse for evaluation purposes. The results are shown in Fig. 12. Here, it is clear that the desired preinverse and postinverse are not the same, and that $S^{\dagger}$ cannot straight away be used as a postinverse. If instead, the output $y$ is filtered by the cascaded systems $1 / G_{m}, S^{\dagger}$ and $G_{m}$, as in Fig. 9, the result improves considerably, as shown in Fig. 12. The remaining errors are primarily caused by the noise. For noise-free data, the preinverse performs perfectly whereas the postinverse has some minor errors.

\section{Inverse estimation}

Hirschorn's method also opens up for questions about how to estimate a system inverse. In the case where the

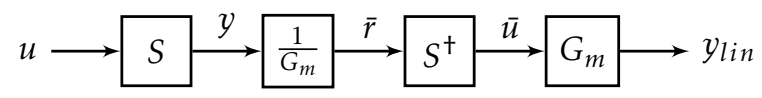

Fig. 10. Hirschorn's method used as postdistortion. The postinverse consists of the three blocks $1 / G_{m}, S^{\dagger}$ and $G_{m}$. Used in this way, the overall behavior between $u$ and $y_{\text {lin }}$ will be linear. 


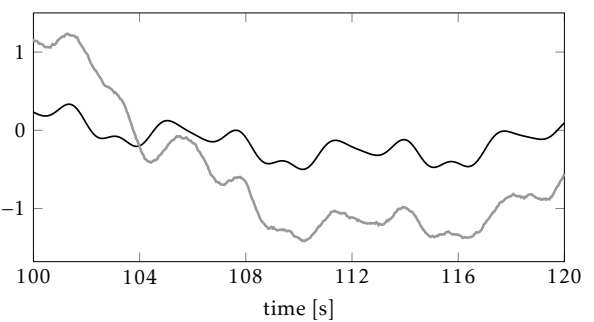

Fig. 11. The output from the nonlinear system (13) in gray and the desired dynamics from $G_{m}$ in black.

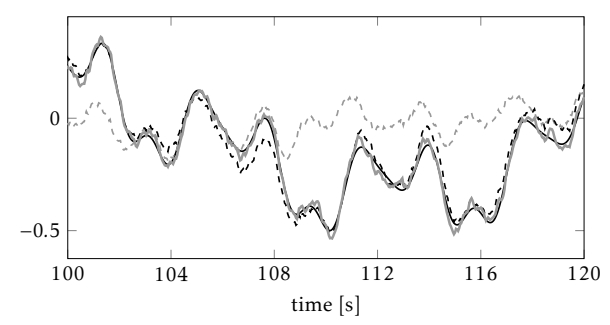

Fig. 12. A Hirschorn postinverse applied to the system (13). The output from $G_{m}$ (solid black) and the output when $S^{\dagger}$ was used as a preinverse (dashed black). The output from the system in series with the inverse $S^{\dagger}$ is plotted in dashed gray when $S^{\dagger}$ is used as a postinverse with no extra filtering. When the postdistortion is constructed according to Fig. 9, the result improves considerably. Here, the postinverse consists of three blocks, $1 / G_{m}, S^{\dagger}$ and $G_{m}$ and the postdistorted output is plotted in solid gray.

structure of the nonlinear system is known, but where there are unknown parameters to be estimated, the identification can be done in several ways, as described in Section II.

Method A would correspond to measuring the input $u$ and the output $y$, and identifying the unknown parameter values in the standard way. This estimated model could then be used to provide the inverse, since if a model of the forward system is available, a model of the inverse system is as well. Method B does not really have an equivalence in this case once the forward model is known, the exact inverse to match it is also known in the exact linearization framework.

Method C would correspond to estimating the inverse $S^{\dagger}$ directly. In order to do this, we would need $u$ as output and the reference $r$ as input. But, as the data was collected in open loop with no pre- or postdistorter, the signal $r$ is not available. Now, as in Section VI-B, assume that the system was actually preceded by a system $S^{\dagger}$, fed by a fictitious reference signal $\tilde{r}$, and that the overall behavior from $\tilde{r}$ to $y$ is in fact linear with dynamics described by $G_{m}$. If this is true, then the signal $\bar{r}$ would be obtained by filtering $y$ with $1 / G_{m}$, and the system $S^{\dagger}$ can be identified using $\bar{r}$ as input and $u$ as output. So, this equals finding the inverse by using (a filtered version of) the output $y$ as the input and $u$ as output. A benefit with Hirschorn's method is that it provides a parameterized inverse, so that the structure of this inverse system is already known.

Estimating inverse models is much harder in the nonlinear dynamical case, and many open questions remain. Still, the discussion on the different methods and the merits and drawbacks of using them is an interesting topic.

\section{CONCLUSIONS}

This paper presents insights and discussions on the estimation of inverse systems. The estimated inverse is intended to be used as a pre- or postdistorter of the original system. A good inverse model is thus one that, when used in series with the original system, reconstructs the original input.

It is shown for the LTI case that the frequency weighting of the estimated inverse model differs, compared to the inverse of an estimated forward model. For a forward model, this contribution will be larger in frequency bands with a large amplification, so that the minimization will focus the model fit to these bands. For the estimation of the inverse, a relative model error will contribute as much to the total loss function, regardless of the amplitude of the transfer function gain (for a white noise input).

For nonlinear systems, one way to obtain a linearizing prefilter is by the use of Hirschorn's algorithm. It is shown how to extend this to the postdistortion case, if the input is unavailable for manipulation. The estimation of this pre- or postinversion model is also discussed.

\section{REFERENCES}

[1] Y. Jung, J. Fritzin, M. Enqvist, and A. Alvandpour, "Least-squares phase predistortion of a $+30 \mathrm{dbm}$ Class-D outphasing RF PA in $65 \mathrm{~nm}$ CMOS," IEEE Trans. Circuits Syst. I, Reg. papers, vol. 60, no. 7, pp. 1915-1928, July 2013.

[2] L. Ljung, System Identification, Theory for the user, 2nd ed. Prentice Hall PTR, 1999.

[3] M. Gevers and L. Ljung, "Optimal experimental designs, with respect to the intended model application," Automatica, vol. 22, no. 5, pp. 543-554, September 1986.

[4] R. Pintelon and J. Schoukens, System Identification, A frequency domain approach, 2nd ed. IEEE Press and John Wiley \& Sons, 2012.

[5] R. M. Hirschorn, "Invertibility of multivariable nonlinear control systems," IEEE Trans. Autom. Control, vol. 24, no. 6, pp. 855-865, December 1979.

[6] K. J. Åström and T. Hägglund, Advanced PID Control, 2nd ed. ISA - Instrumentation, Systems, and Automation Society, 2005.

[7] S. Devasia, "Should model-based inverse inputs be used as feedforward under plant uncertainty," IEEE Trans. Autom. Control, vol. 47, no. 11, pp. 1865-1871, November 2002.

[8] J. Fritzin, Y. Jung, P. N. Landin, P. Händel, M. Enqvist, and A. Alvandpour, "Phase predistortion of a class-D outphasing RF amplifier in 90nm CMOS," IEEE Trans. Circuits Syst. II, Exp. Briefs, vol. 58, no. 10, pp. 642-646, October 2011.

[9] E. Abd-Elrady, L. Gan, and G. Kubin, "Direct and indirect learning methods for adaptive predistortion of IIR Hammerstein systems," Elektrotechnik \& Informationstechnik, vol. 125, no. 4, pp. 126-131, April 2008.

[10] H. Paaso and A. Mämmelä, "Comparison of direct learning and indirect learning predistortion architechtures," in IEEE Int. Syp. Wireless Comm. Systems (ISWCS), October 2008, pp. 309-313.

[11] R. Vuerinckx, Y. Rolain, J. Schoukens, and R. Pintelon, "Design of stable IIR filters in the complex domain by automatic delay selection," IEEE Transactions on Signal Processing, vol. 44, no. 9, pp. 23392344, September 1996.

[12] R. Pintelon, Y. Rolain, M. V. Bossche, and J. Schoukens, "Towards an ideal data acquisition channel," IEEE Trans. Instrum. Meas., vol. 39, no. 1, pp. 116-120, February 1990.

[13] K. J. Åström and P. Eykhoff, "System identification - A survey," Automatica, vol. 7, pp. 123-162, 1971.

[14] O. Markusson, "Model and system inversion with applications in nonlinear system identification and control," TRITA-S3-REG-0201, Royal Institute of Technology, Stockholm, Sweden, 2001.

[15] M. Schetzen, The Volterra and Wiener Theories of Nonlinear Systems. John Wiley \& Sons, New York, 1980.

[16] S. Sastry, Nonlinear Systems - Analysis, Stability and Control. Springer Verlag, New York, 1999. 